IFIC $/ 97-98$

\title{
Translationally-invariant coupled-cluster method for finite systems
}

\author{
R. Guardiola, I. Moliner \\ Dept. de Física Atómica, Molecular i Nuclear, Universitat de València \\ Avda. Dr. Moliner 50, E-46.100 Burjassot, Spain \\ J. Navarro, M. Portesi \\ IFIC (Centre Mixt CSIC - Universitat de València) \\ Avda. Dr. Moliner 50, E-46.100 Burjassot, Spain
}

\begin{abstract}
The translational invariant formulation of the coupled-cluster method is presented here at the complete $\mathrm{SUB}(2)$ level for a system of nucleons treated as bosons. The correlation amplitudes are solution of a non-linear coupled system of equations. These equations have been solved for light and medium systems, considering the central but still semi-realistic nucleon-nucleon S3 interaction.
\end{abstract}

PACS: 21.60.-n; 31.15.Dv

Keywords: Many-body techniques; two-body correlations; coupled-cluster methods 


\section{Introduction}

The coupled-cluster method (CCM) was invented many years ago by Coester and Kümmel 11, 21 to calculate the ground-state energy of closed-shell nuclei. Since then the CCM has been developed for applications in a wide variety of fields, including quantum chemistry, quantum lattices, electron gas, or nuclear physics. Although the formalism was initially developed to calculate only the energy of the ground state of a closed-shell nucleus, the CCM is not just a theory for the ground state of closed-shell systems. Further extensions allow to calculate also excited states, open-shell systems, or finite temperature systems, to mention only a few developments of CCM (see general references in Refs. [3, 4). Currently, the CCM provides a widely applicable formalism to deal with the general quantum manybody problem at a completely microscopic level. It works for systems of both bosons and fermions, regardless of the type and range of the interaction.

The CCM wave function is described in terms of the action of a cluster operator on some reference function, which takes into account the required symmetry properties of the system under consideration. The cluster operator is sum of $n$-body operators, which create $n$ particle-hole pairs on the reference function. In the application to finite systems, one faces with the well-known center-of-mass motion problem. The proper treatment of the center-of-mass in the framework of CCM was initiated some years ago [5, 6] at the so-called SUB(2) level of approximation, which is limited up to two-body operators. It has been shown in these references that the center-of-mass is properly removed using appropriate combinations of one- and two-body operators, and describing the reference function in terms of single-particle harmonic oscillator wave functions. Such reformulation of the CCM is called translationally invariant coupled-cluster (TICC) method, and it has been applied to the ${ }^{4} \mathrm{He}$ nucleus, considered as a system of four bosons. For the simple interactions considered in Ref. [6], it was shown that the results at the SUB(2) level (TICC2 henceforth) may differ in a few MeV from the essentially exact diffusion Monte Carlo results.

The CCM is most naturally formulated in the occupation number representation. However, very slow convergence with respect to the cut-off on the single particle basis has been found. This led to consider a linearized form of the TICC2 approach, which can be easily converted to coordinate representation. The pair correlations are considered through a function depending on the relative coordinate of a single pair. The ansatz for the wave function of a fermionic system is

$$
|\Psi\rangle=\sum_{i<j}\left(f_{c}\left(r_{i j}\right)+f_{\sigma}\left(r_{i j}\right)\left(\sigma_{i} \cdot \sigma_{j}\right)+f_{\tau}\left(r_{i j}\right)\left(\tau_{i} \cdot \tau_{j}\right)+f_{\sigma \tau}\left(r_{i j}\right)\left(\sigma_{i} \cdot \sigma_{j}\right)\left(\tau_{i} \cdot \tau_{j}\right)\right)|\Phi\rangle,
$$

where $|\Phi\rangle$ is the reference state. The four unknown two particle correlation functions may be determined by minimizing the expectation value of the hamiltonian, and a single correlation function appears in the case of a system of bosons. This so-called translationally invariant configuration interaction method was applied in Ref. [7] to calculate at the $\operatorname{SUB}(2)$ level (or TICI2 approach) the ground-state energy of some light nuclei in the $1 p$-shell up to ${ }^{16} \mathrm{O}$. The main conclusion of this work is that the TICI2 methodology provides a very reasonable starting point for the calculation of the binding energies of light-to-medium nuclei. For interactions and correlations of the V4 form, as displayed in 
the above equation, the TICI2 results are in suitable agreement with the ones provided by other methodologies.

In order to get a better description of finite nuclei there are however some obvious extensions to be performed. Among them are the inclusion of all the correlations at the SUB(2) level, i.e. the TICC2 approximation. In Refs. [5, 6] it has been shown that the additional terms give a relatively small contribution to the ground-state energy of the ${ }^{4} \mathrm{He}$ nucleus. The purpose of the present work is to discern the importance of the nonlinear terms beyond TICI2 in the description of a bosonic system. In Section 2 we briefly summarize the main findings of previous works [5, 6], generalizing at the same time the results for $N$ bosons. In Section 3 the detailed equations are presented and discussed. An application example is given in Section 4, where a simple nuclear interaction is used to determine the ground-state energy of a system of nucleons treated as bosons. Finally, in Section 5 some concluding remarks and an outlook for further studies are given.

\section{The Translationally Invariant SUB(2) approximation for bosons}

The basic CCM ansatz is to write the $N$-body wave function as

$$
|\Psi\rangle=\mathrm{e}^{S}|\Phi\rangle,
$$

where $|\Phi\rangle$ is a reference state, incorporating the statistical and symmetry properties of the system, and

$$
S=\sum_{n=1}^{N} S_{n}
$$

is the cluster correlation operator, sum of operators of the form

$$
S_{n}=\frac{1}{(n !)^{2}} \sum_{\rho_{1}, \ldots, \rho_{n}}\left\langle\rho_{1}, \ldots, \rho_{n}\left|S_{n}\right| 0, \ldots, 0\right\rangle a_{\rho_{1}}^{+} \ldots a_{\rho_{n}}^{+} a_{0}^{n},
$$

whose action is to create $n$ particle-hole excitations on the reference function. The $\mathrm{SUB}(2)$ level approximation of CCM consists of neglecting the excitations of more than two particle-hole pairs, so that in the initial ansatz one has $S=S_{1}+S_{2}$.

In order to get rid of the center-of-mass problem, one could directly choose a translationally invariant reference state, but this will spoil the meaning of the Fock space operator as single-particle operators, due to the center-of-mass constraint. The alternative followed in Ref. [5] was to build up the reference state from harmonic-oscillator (HO) single-particle wave functions, which can be defined through creation operators acting on the vacuum,

$$
|n l m\rangle=a_{n l m}^{+}|0\rangle,
$$

and to write the reference state of an $N$-boson system as

$$
|\Phi\rangle=\frac{1}{\sqrt{N !}}\left(a_{000}^{+}\right)^{N}|0\rangle .
$$


It is well-known that this $\mathrm{HO}$ uncorrelated wave function is the simple product of a $1 s \mathrm{HO}$ wave function for the center-of-mass and an intrinsic wave function. Although the reference state is not translationally invariant, this factorization property allows one to remove the center-of-mass motion unambiguously. Dressing the reference state with correlations has the danger of spoiling the factorization property, unless the correlations are translationally invariant. Therefore our immediate goal is to construct excitations on $|\Phi\rangle$ without exciting the center-of-mass, so that in this way it can be properly removed from the SUB(2) wave function.

With only $S_{1}$ cluster operators it is not possible to get translationally invariant correlations. However, appropriate admixtures of $S_{1}$ and $S_{2}$ can do it. Let us indicate by $S^{(1,2)}$ the corresponding new cluster operator. Translational invariance may be imposed by: i) recoupling the product of single-particle harmonic-oscillator states into sums of comparable products of oscillator states for the relative and center-of-mass motion of the pair, and ii) imposing that the center-of-mass state of the destroyed pair of particles in the occupied subspace is the same as that of the created pair of particles in the unoccupied subspace. Since we are using $\mathrm{HO}$ wave functions, this is accomplished by using the Brody-Moshinsky brackets [8]. In the case of an $N$ boson system, one can write the cluster operator as

$$
S^{(1,2)}=\sum_{n=1}^{\infty} \mathcal{S}(n) \sum_{n_{1}, n_{2}, l}\left\langle n 0,00,0 \mid n_{1} l, n_{2} l, 0\right\rangle\left[a_{n_{1} l}^{+} \times a_{n_{2} l}^{+}\right]_{0}^{0} a_{000}^{2},
$$

where $\left\langle n 0,00,0 \mid n_{1} l, n_{2} l, 0\right\rangle$ is a Brody-Moshinsky coefficient, which limits the sums to the cases $n_{1}+n_{2}+l=n$. This expression reflects that two bosons in occupied states of $|\Phi\rangle$ are destroyed, and the created pair of particles has zero angular momentum, its centerof-mass being in the $\mathrm{HO}$ state with all quantum numbers equal to zero. Note that the term with both $\left(n_{1}, l\right)=(0,0)$ and $\left(n_{2}, l\right)=(0,0)$ is excluded, as it simply reproduces the uncorrelated state $|\Phi\rangle$. However, the terms with either $\left(n_{1}, l\right)=(0,0), n_{2} \neq 0$ or $n_{1} \neq 0,\left(n_{2}, l\right)=(0,0)$ are included. These terms give precisely the $1 \mathrm{p}-1 \mathrm{~h}$ excitations in the admixture.

It is worth noting the enormous simplification imposed by the translational invariance, because we have a single correlation amplitude $\mathcal{S}(n)$, whose argument is just a single number which counts the number of oscillator quanta (divided by 2 ) globally excited. By contrast, in the unrestricted $\operatorname{SUB}(2)$ approximation, one deals with two correlation amplitudes $\mathcal{S}_{1}(n)$ and $\mathcal{S}_{2}\left(n_{1}, n_{2}, l\right)$.

It is convenient to use the following simplified notation for the correlation operator:

$$
S^{(1,2)}=s(p) a_{p}^{+} a_{0}^{+} a_{0}^{2}+s(p, q) a_{p}^{+} a_{q}^{+} a_{0}^{2},
$$

where a sum over the particle indices $p, q$ is to be assumed. These indices refer to the HO quantum numbers: $p \equiv\left(n_{p}, l_{p}, m_{p}\right)$, and they are different from $(0,0,0)$, since they correspond to non-occupied states. For convenience, we shall also use the notation $0 \equiv$ $(0,0,0)$. The coefficients are defined as

$$
\begin{aligned}
s(p)= & \delta\left(l_{p}, 0\right) \delta\left(m_{p}, 0\right) 2\left\langle n_{p} 0,00,0 \mid n_{p} 0,00,0\right\rangle \mathcal{S}\left(n_{p}\right), \\
s(p, q)= & \delta\left(l_{p}, l_{q}\right) \delta\left(m_{p},-m_{q}\right) C\left(l_{p}, l_{p}, 0 ; m_{p},-m_{p}, 0\right) \\
& \left\langle n_{p}+n_{q}+l_{p} 0,00,0 \mid n_{p} l_{p}, n_{q} l_{p}, 0\right\rangle \mathcal{S}\left(n_{p}+n_{q}+l_{p}\right),
\end{aligned}
$$


where $C\left(l_{1}, l_{2}, l_{3} ; m_{1}, m_{2}, m_{3}\right)$ is a Clebsh-Gordan coefficient.

Finally, let us note that simply because $S^{(1,2)}$ is invariant under translations we are by no means guaranteed that so does the exponential form $\mathrm{e}^{S^{(1,2)}}$. This can be easily seen by considering the simple example of the squared correlation operator $S^{(1,2)} S^{(1,2)}$. Indeed, one of the destruction operators $a_{0}$ of one factor may be contracted with the creation operator $a_{0}^{+}$of the other factor, resulting in a term which is not translationally invariant. As was shown in Ref. [5] the unwanted terms are easily excluded by the simple device of

taking the $\mathrm{e}^{S^{(1,2)}}$ operator in normal-ordered form, so that the TICC2 ansatz for the wave function can be finally written as

$$
|\Psi\rangle=: \mathrm{e}^{S^{(1,2)}}:|\Phi\rangle
$$

In principle, for a system of bosons the normal ordered form does not imply a particular technical problem (contrary to the fermionic case). However, the usual CCM equations are more involved, and we found it more convenient to derive the CCM equations in a different manner, as it will be shown in the next section.

\section{The TICC2 equations}

The usual way to solve the Schrödinger equation

$$
H|\Psi\rangle=E|\Psi\rangle
$$

within CCM involves transforming it first to

$$
\mathrm{e}^{-S} H \mathrm{e}^{S}|\Phi\rangle=E|\Phi\rangle,
$$

i.e. to define a similarity transformed hamiltonian. The motivation to use this equation is that the transformed hamiltonian $\mathrm{e}^{-S} \mathrm{He}^{S}$ may be reduced to a set of nested commutators, which may result in a simplification of the algebra. Afterwards, Eq. (12) is projected onto $0 \mathrm{p}-0 \mathrm{~h}, 1 \mathrm{p}-1 \mathrm{~h}, \ldots$ states to get algebraic equations for the amplitudes. If we were projecting up to $N \mathrm{p}-N \mathrm{~h}$, the corresponding set of algebraic equations would be equivalent to the Schrödinger equation. However, if the cluster operator is truncated at a given $\operatorname{SUB}(n)$ level the projection up to $N \mathrm{p}-N$ h states will produce redundant equations, so the current practice is to project up to the $n \mathrm{p}-n$ h states. It is worth noting that if the cluster operator is truncated at the $\operatorname{SUB}(n)$ level none of Eqs. (11) or (12) may be exactly satisfied, and moreover one should not expect in general to obtain the same results from both equations.

The similarity transformation does not appear convenient in our formalism with the normal ordering prescription on $\exp (S)$. Certainly, the inverse of the operator : $\exp (S)$ : is not $: \exp (-S):$, but it is possible to find a new operator $T$ such that the equation

$$
T: \mathrm{e}^{S}:|\Phi\rangle=|\Phi\rangle
$$

is satisfied. For the specific operator $S^{(1,2)}$ given in Eq. (đ), there results

$$
T \simeq 1-(N-1) s(p) a_{p}^{+} a_{0}+\left[-s(p, q)+\frac{1}{2}(N+1)(N-2) s(p) s(q)\right] a_{p}^{+} a_{q}^{+} a_{0}^{2}+\ldots,
$$


up to two creation operators. This operator $T$ is not the inverse of : $\exp \left(S^{(1,2)}\right)$ : because it is defined when acting on the reference state $|\Phi\rangle$, and this fact is reflected in its dependence on the number of bosons of the system. The structure of $T$ depends on the reference state, because also normal ordering depends on such state.

Another much simpler alternative to obtain the equations for the amplitudes is to project directly the Schrödinger equation with the ansatz (10)

$$
H: \mathrm{e}^{S^{(1,2)}}:|\Phi\rangle=E: \mathrm{e}^{S^{(1,2)}}:|\Phi\rangle
$$

onto the states with $0 \mathrm{p}-0 \mathrm{~h}, 1 \mathrm{p}-1 \mathrm{~h}$ and $2 \mathrm{p}-2 \mathrm{~h}$.

For hamiltonians with two-body interaction potentials like

$$
H=\sum_{\alpha, \beta}\langle\alpha|K| \beta\rangle a_{\alpha}^{+} a_{\beta}+\frac{1}{4} \sum_{\alpha_{1}, \alpha_{2}, \beta_{1}, \beta_{2}}\left\langle\alpha_{1}, \alpha_{2}|V| \beta_{1}, \beta_{2}\right\rangle a_{\alpha_{1}}^{+} a_{\alpha_{2}}^{+} a_{\beta_{2}} a_{\beta_{1}}
$$

one requires the expansion of : $\exp \left(S^{(1,2)}\right)$ : up to terms involving at most four particle creation operators. In terms of the quantities $s(p)$ and $s(p, q)$ defined in Eqs. (8) and (9), respectively, one may effectively do the substitution

$$
\begin{aligned}
: \mathrm{e}^{S^{(1,2)}:=} & 1+s(p) a_{p}^{+} a_{0}^{+} a_{0}^{2}+s(p, q) a_{p}^{+} a_{q}^{+} a_{0}^{2}+\frac{1}{2} s(p) s(q) a_{p}^{+} a_{q}^{+}\left(a_{0}^{+}\right)^{2} a_{0}^{4} \\
& +s(p) s(q, r) a_{p}^{+} a_{q}^{+} a_{r}^{+} a_{0}^{+} a_{0}^{4}+\frac{1}{6} s(p) s(q) s(r) a_{p}^{+} a_{q}^{+} a_{r}^{+}\left(a_{0}^{+}\right)^{3} a_{0}^{6} \\
& +\frac{1}{2} s(p, q) s(r, s) a_{p}^{+} a_{q}^{+} a_{r}^{+} a_{s}^{+} a_{0}^{4}+\frac{1}{2} s(p) s(q) s(r, s) a_{p}^{+} a_{q}^{+} a_{r}^{+} a_{s}^{+}\left(a_{0}^{+}\right)^{2} a_{0}^{6} \\
& +\frac{1}{24} s(p) s(q) s(r, s) a_{p}^{+} a_{q}^{+} a_{r}^{+} a_{s}^{+}\left(a_{0}^{+}\right)^{4} a_{0}^{8}+\ldots,
\end{aligned}
$$

which is no more complex than the usual nested commutators. Note the reorderings of $a_{0}$ and $a_{0}^{+}$related to the normal ordering prescription.

The projection onto $0 \mathrm{p}-0 \mathrm{~h}$ is simply the projection onto the bra state $\langle\Phi|$ defined by the reference state, and it gives the ground-state energy:

$$
E=\left\langle\Phi\left|H: \mathrm{e}^{S^{(1,2)}}:\right| \Phi\right\rangle .
$$

Using the following notation:

$$
\begin{aligned}
k(\alpha, \beta) & =\left\langle n_{\alpha} l_{\alpha} m_{\alpha}|K| n_{\beta} l_{\beta} m_{\beta}\right\rangle, \\
v\left(\alpha_{1}, \alpha_{2}, \beta_{1}, \beta_{2}\right) & =\left\langle n_{\alpha_{1}} l_{\alpha_{1}} m_{\alpha_{1}} ; n_{\alpha_{2}} l_{\alpha_{2}} m_{\alpha_{2}}|V| n_{\beta_{1}} l_{\beta_{1}} m_{\beta_{1}} ; n_{\beta_{2}} l_{\beta_{2}} m_{\beta_{2}}\right\rangle,
\end{aligned}
$$

for the matrix elements of kinetic and potential energies taken between $\mathrm{HO}$ single particle wave functions, we find

$$
\begin{aligned}
E & =N_{1} k(0,0)+N_{2} v(0,0,0,0) \\
& +N_{2}[k(0, p) s(p)+2(N-1) v(0,0, p, 0) s(p)+2 v(0,0, p, q) s(p, q)] \\
& +N_{4} v(0,0, p, q) s(p) s(q),
\end{aligned}
$$


where we have also defined

$$
N_{i}=N(N-1) \ldots(N-i+1)
$$

Again, a sum over repeated particle indices $p, q$ is to be understood in Eq. (21).

The ground-state energy is thus given in terms of the correlation amplitudes $\mathcal{S}(n)$ implicit in $s(p)$ and $s(p, q)$. To determine these amplitudes, we must project the Schrödinger equation onto $1 \mathrm{p}-1 \mathrm{~h}$ and $2 \mathrm{p}-2 \mathrm{~h}$ states taking care that the center-of-mass state is the same as in the reference function. The obvious state to project on is the appropriate $1 \mathrm{p}-1 \mathrm{~h}$ and $2 \mathrm{p}-2 \mathrm{~h}$ admixture which maintains the factorizability of the center-of-mass in the reference state. Such an admixture is already known, after the discussion of Section 2: it must have the same structure as $S^{(1,2)}$, but without the unknown amplitudes $\mathcal{S}(n)$. It seems convenient to classify the excitations in terms of the number of oscillator quanta $2 N_{x}$, where $N_{x}$ will run from 1 up to some sufficiently high value $N_{\max }$ so as to reach convergence. In this way one obtains $N_{\max }$ non-linear equations to determine the unknown amplitudes $\mathcal{S}(n)$. Once these amplitudes are known, the energy is easily computed.

Consequently, the excited bra state required for the proper projection can be written as

$$
\langle\Phi|\left[c(m)\left(a_{0}^{+}\right)^{2} a_{0} a_{m}+c(m, n)\left(a_{0}^{+}\right)^{2} a_{m} a_{n}\right],
$$

where the excitation amplitudes have a structure similar to the cluster operator, that is

$$
\begin{gathered}
c(m)=\delta\left(l_{m}, 0\right) \delta\left(m_{m}, 0\right) 2\left\langle N_{x} 0,00,0 \mid n_{m} 0,00,0\right\rangle \\
c(m, n)=\delta\left(l_{m}, l_{n}\right) \delta\left(m_{m},-m_{n}\right) C\left(l_{m}, l_{m}, 0 ; m_{m},-m_{m}, 0\right)\left\langle N_{x} 0,00,0 \mid n_{m} l_{m}, n_{n} l_{m}, 0\right\rangle .
\end{gathered}
$$

The indices $m, n$ refer to excited states, and cannot be identical with $(0,0,0)$. Moreover, they are external indices, tied to the excitation quantum number $N_{x}$, so that there is no summation over them.

Thus, the equation to determine the correlation amplitudes can be written as:

$$
\begin{aligned}
& \left\langle\Phi\left|\left[c(m)\left(a_{0}^{+}\right)^{2} a_{0} a_{m}+c(m, n)\left(a_{0}^{+}\right)^{2} a_{m} a_{n}\right] H: \mathrm{e}^{S^{(1,2)}}:\right| \Phi\right\rangle \\
& \quad=E\left\langle\Phi\left|\left[c(m)\left(a_{0}^{+}\right)^{2} a_{0} a_{m}+c(m, n)\left(a_{0}^{+}\right)^{2} a_{m} a_{n}\right]: \mathrm{e}^{S^{(1,2)}}:\right| \Phi\right\rangle .
\end{aligned}
$$

Evaluating all contractions contained in this equation is not a simple task, because of the very large number of terms which appear after the expansion of the operators. We have found of great help the use of the algebraic language REDUCE円. The core of the evaluation is the substitution

$$
a_{x} a_{y}^{+} \rightarrow a_{y}^{+} a_{x}+\delta(x, y)
$$

which is controlled by a let rule. This automatically reorders the full expression with all annihilation operators on the right. Afterwards the Kronecker delta functions are conveniently eliminated.

\footnotetext{
${ }^{1}$ Copyright (C)1993 The RAND Corporation, Santa Monica, CA, USA.
} 
The left-hand side of Eq. (26) can be written in the form

$$
\begin{aligned}
& \left\langle\Phi\left|\left[c(m)\left(a_{0}^{+}\right)^{2} a_{0} a_{m}+c(m, n)\left(a_{0}^{+}\right)^{2} a_{m} a_{n}\right] H: \mathrm{e}^{S^{(1,2)}}:\right| \Phi\right\rangle \\
& =N_{2} c(m) k(m, 0) \\
& +\quad N_{2}\left[2(N-2) c(m, n) k(0,0) s(m, n)+(N-1)^{2} c(m) k(0,0) s(m)\right. \\
& +2(N-1) c(m, n) k(m, 0) s(n)+2(N-1) c(m) k(0, p) s(m, p) \\
& +4 c(m, n) k(m, p) s(n, p)+(N-1) c(m) k(m, p) s(p)] \\
& +\quad N_{4}[(N-2) c(m, n) k(0,0) s(m) s(n)+2 c(m, n) k(0, p) s(m, n) s(p) \\
& +4 c(m, n) k(0, p) s(m, p) s(n)+(N-1) c(m) k(0, p) s(m) s(p) \\
& +2 c(m, n) k(m, p) s(n) s(p)] \\
& +\quad N_{6}[c(m, n) k(0, p) s(m) s(n) s(p)] \\
& +\quad N_{2}[2(N-1) c(m) v(m, 0,0,0)+2 c(m, n) v(m, n, 0,0)] \\
& +\quad N_{2}\left[(N-1)^{2}(N-2) c(m) v(0,0,0,0) s(m)\right. \\
& +2(N-2)(N-3) c(m, n) v(0,0,0,0) s(m, n) \\
& +4(N-1)(N-2) c(m, n) v(m, 0,0,0) s(n) \\
& +4(N-1)(N-2) c(m) v(0,0, p, 0) s(m, p) \\
& +4(N-1)^{2} c(m) v(m, 0, p, 0) s(p)+16(N-2) c(m, n) v(n, 0, p, 0) s(m, p) \\
& +4(N-1) c(m) v(m, 0, p, q) s(p, q)+4(N-1) c(m, n) v(m, n, p, 0) s(p) \\
& +4 c(m, n) v(m, n, p, q) s(p, q)] \\
& +\quad N_{4}[(N-2)(N-3) c(m, n) v(0,0,0,0) s(m) s(n) \\
& +4(N-3) c(m, n) v(0,0, p, 0) s(m, n) s(p) \\
& +8(N-3) c(m, n) v(0,0, p, 0) s(m, p) s(n) \\
& +2(N-1)(N-2) c(m) v(0,0, p, 0) s(m) s(p) \\
& +4 c(m, n) v(0,0, p, q) s(m, n) s(p, q)+8 c(m, n) v(0,0, p, q) s(m, p) s(n, q) \\
& +8(N-2) c(m, n) v(n, 0, p, 0) s(m) s(p)+4(N-1) c(m) v(0,0, p, q) s(m, q) s(p) \\
& +2(N-1) c(m) v(0,0, p, q) s(m) s(p, q)+2(N-1) c(m) v(m, 0, p, q) s(p) s(q) \\
& +16 c(m, n) v(n, 0, p, q) s(m, q) s(p)+8 c(m, n) v(n, 0, p, q) s(m) s(p, q) \\
& +2 c(m, n) v(m, n, p, q) s(p) s(q)] \\
& +\quad N_{6}[2(N-3) c(m, n) v(0,0, p, 0) s(m) s(n) s(p) \\
& +(N-1) c(m) v(0,0, p, q) s(m) s(p) s(q) \\
& +8 c(m, n) v(0,0, p, q) s(m, p) s(n) s(q)+2 c(m, n) v(0,0, p, q) s(m, n) s(p) s(q)
\end{aligned}
$$




$$
\begin{aligned}
& \quad+2 c(m, n) v(0,0, p, q) s(m) s(n) s(p, q)+4 c(m, n) v(n, 0, p, q) s(m) s(p) s(q)] \\
& +\quad N_{8}[c(m, n) v(0,0, p, q) s(m) s(n) s(p) s(q)] .
\end{aligned}
$$

In the right-hand side of Eq. (26) one has

$$
\begin{aligned}
& \left\langle\Phi\left|\left[c(m)\left(a_{0}^{+}\right)^{2} a_{0} a_{m}+c(m, n)\left(a_{0}^{+}\right)^{2} a_{m} a_{n}\right]: \mathrm{e}^{S^{(1,2)}}:\right| \Phi\right\rangle \\
= & N_{2}[(N-1) c(m) s(m)+2 c(m, n) s(m, n)] \\
+ & N_{4}[c(m, n) s(m) s(n)] .
\end{aligned}
$$

Let us recall that in these equations any index $m, n, p$, or $q$ must be interpreted as a set of three quantum numbers $n \equiv\left(n_{n}, l_{n}, m_{n}\right)$ of the HO states, none of them being $(0,0,0)$. Let us insist again that in these expressions, indices $p, q$ refer to particle states and a sum on them must be understood, indices $m, n$ are external, and are related to the quantum number $N_{x}$ characterizing the excitation.

We have stressed the factors $N_{2}, N_{4}, N_{6}$, and $N_{8}$ accompanying the correlation of one, two, three, and four particle-hole pairs. The results of Ref. [5] showed that in the case of ${ }^{4} \mathrm{He}$ the quadratic terms give a relatively unimportant contribution to the ground-state energy, but we cannot assume this to be the case for larger systems. Firstly, it is clear that only two pairs can be created for $N=4$ particles, and consequently the TICC2 approximation for ${ }^{4} \mathrm{He}$ contains only linear and quadratic terms. But more pairs can be excited for $N>4$, and we see that up to four pairs are present when the interaction is a two-body one. Secondly, the expressions given above for the projection show that these terms are multiplied by increasing powers of $N$ : quadratic terms are multiplied by a factor $N_{4}$, cubic terms by $N_{6}$, and quartic terms by $N_{8}$. Although the amplitudes $\mathcal{S}(n)$ may depend on $N$ one cannot discard the possibility that the non-linear terms could become relatively more important for high values of $N$.

We have to solve a non-linear (actually quartic) system of coupled equations to determine the amplitudes $\mathcal{S}(n)$, which can be cast in the following schematic form:

$$
\sum_{i_{1}, i_{2}, i_{3}, i_{4}=0}^{N_{\max }} F\left(N_{x}, i_{1}, i_{2}, i_{3}, i_{4}\right) \mathcal{S}\left(i_{1}\right) \mathcal{S}\left(i_{2}\right) \mathcal{S}\left(i_{3}\right) \mathcal{S}\left(i_{4}\right)=0
$$

with the convention $\mathcal{S}(0)=1$. The number $N_{x}=1, \ldots, N_{\max }$ refers to the excitation considered. The function $F\left(N_{x}, i_{1}, i_{2}, i_{3}, i_{4}\right)$ is obtained from the expressions given above for the projections onto the excitations up to $2 N_{x}$ quanta. The indices $i$ in the correlation amplitudes refer to the number $2 i$ of $\mathrm{HO}$ quanta characterizing the excitation, which we limit to a maximum number $N_{\max }$. The independent term is given by $F\left(N_{x}, 0,0,0,0\right)$. The linear version of the present development, or TICI2 approximation, amounts to consider in Eq. (30) only the function $F\left(N_{x}, i_{1}, 0,0,0\right)$ (including $i_{1}=0$ ), and the problem is reduced to solve a generalized eigenvalue equation.

There is still a long way from Eqs. (28) - (29) to the practical equations. Rotational invariance of the hamiltonian merged with the specific form of $s(p)$ and $s(p, q)$ results in a 
drastic reduction on the number of summed up indices. Additionally, the potential matrix elements are conveniently transformed into Talmi integrals, referring only to the relative coordinate of the pair. The final formula is too long to be included here. A part of the non-linear equations, up to quadratic terms in the amplitudes, may be found in Appendix A of Ref. [5], for the $N=4$ case.

\section{An application example}

We consider a model system consisting of $N$ nucleons treated as bosons interacting via the Wigner part of a nuclear interaction. These systems collapse for large values of $N$, and so one could reasonably expect that the effects related to the correlations will be enhanced. We have used the Wigner part of the Afnan-Tang nucleon-nucleon potential S3 [9], which is considered as a semi-realistic interaction having a quite strong core at the origin. This will enhance again the role of the correlations.

The price to be paid when going from the configuration interaction TICI2 scheme to the full TICC2 one is that instead of solving a generalized eigenvalue problem as was done in Ref. [7], one has to solve the non-linear system of equations (30) coupled to the equation determining the energy $E=\left\langle\Phi\left|H: \exp \left(S^{(1,2)}\right):\right| \Phi\right\rangle$. The obvious steps are first to obtain explicitly the matrix of coefficients $F\left(N_{x}, i_{1}, i_{2}, i_{3}, i_{4}\right)$ and afterwards to solve for the amplitudes and the energy. The equations depend on the harmonic oscillator parameter $\alpha=(m \omega / \hbar)^{1 / 2}$ used to define the Fock space, so it is convenient to adjust this $\hbar \omega$ parameter like in a variational calculation.

As we have already mentioned, we may limit the number of equations by means of the index $N_{\text {max }}$, which measures the number of excitation quanta $\left(2 N_{\max } \hbar \omega\right)$ related to a given term of the correlation. One may then proceed sequentially, starting from a small value of $N_{\max }$, and taking its solution as the approximate solution corresponding to the next set

of $N_{\max }+1$ equations. The Newton-Raphson method turns out to be very efficient once we are quite close to the solution.

An alternative way is to introduce in Eq. (30) a quenching factor $q$ as follows:

$$
\begin{aligned}
& \sum_{i_{1}=0}^{N_{\max }} F\left(N_{x}, i_{1}, 0,0,0\right) \mathcal{S}\left(i_{1}\right) \\
+ & q \sum_{i_{1}=0}^{N_{\max }} \sum_{\left(i_{2}, i_{3}, i_{4}\right) \neq(0,0,0)}^{N_{\max }} F\left(N_{x}, i_{1}, i_{2}, i_{3}, i_{4}\right) \mathcal{S}\left(i_{1}\right) \mathcal{S}\left(i_{2}\right) \mathcal{S}\left(i_{3}\right) \mathcal{S}\left(i_{4}\right)=0,
\end{aligned}
$$

where in the second sum the indices $\left(i_{2}, i_{3}, i_{4}\right)$ cannot be simultaneously equal to zero, as this case is included in the first sum. We let the quenching factor $q$ slowly grow from 0 to 1 , in such a manner that $q=0$ corresponds to the linear problem and $q=1$ corresponds to the full quartic set of equations. Solutions of the full equation are thus smoothly connected to solutions of the simpler linear problem.

There is no a priori way of knowing if all solutions have been determined, as well as which of these solutions are physically relevant. In the TICI2 formulation there are as many solutions as the number of equations; the lowest one corresponds to the ground 
state of the system and the rest could be interpreted as monopole excitations or breathing modes.

We have solved the non-linear system of equations for $N=4,16$ and 40 bosons using the S3 interaction. In Fig. 1 are displayed the energies of two selected TICC2 solutions (only one for $N=4$ ) and their evolution with the sequential scheme. These two solutions are represented by the solid and the long-dashed lines. The linear TICI2 solution is also displayed as the short-dashed line. Several comments are in order regarding these three solutions.

(i) All of them tend to stability with increasing values of $N_{\max }$, and at $N_{\max }=30$ they have already converged.

(ii) There is a connection between the solid line and the TICI2 line in the quenching scheme and in both directions. This means that starting from TICI2 solution $(q=0)$ and slowly increasing the value of $q$ one arrives to the solid line TICC2 solution when $q=1$, and conversely, starting from the solid line TICC2 solution $(q=1)$ one arrives to the TICI2 solution by slowly decreasing $q$ down to $q=0$.

(iii) There is not a connection path which starts from the long-dashed TICC2 solution. When $q$ is slowly decreased the solution disappears.

In conclusion, even if the TICC2 solution represented by the long-dashed line has the lowest energy value, it should be considered as a spurious solution of the TICC2 method which has no relation with the physical problem. This discussion has been quite simplified because we have focused only on two cases. Actually, by generating at random starting points for the Newton-Raphson method, there appears a very large number of solutions which must be discriminated.

The arguments of correctness of solutions, both to increasing $N_{\max }$ or along the quenched path, do not really ascertain the goodness of a given solution. A precise but costly way is to compute the expectation value of the hamiltonian for any of the found solutions, but this is a hopeless task in the Fock representation when a large number of particles is involved. For this calculation one requires the matrix element

$$
\langle H\rangle=\frac{\left\langle\Phi\left|\left(: \mathrm{e}^{S^{(1,2)}}:\right)^{+} H: \mathrm{e}^{S^{(1,2)}}:\right| \Phi\right\rangle}{\left\langle\Phi\left|\left(: \mathrm{e}^{S^{(1,2)}}:\right)^{+}: \mathrm{e}^{S^{(1,2)}}:\right| \Phi\right\rangle},
$$

with a number of contractions scaling as $N^{2}$.

An alternative way is to go to coordinate representation, where

$$
\begin{aligned}
\left\langle\mathbf{r}_{1}, \ldots, \mathbf{r}_{N}\left|: \mathrm{e}^{S^{(1,2)}}:\right| \Phi\right\rangle & =\left\{1+\sum_{i<j} f_{i j}+\frac{1}{2 !} \sum_{i<j} \sum_{k<l}{ }^{\prime} f_{i j} f_{k l}\right. \\
& \left.+\frac{1}{3 !} \sum_{i<j} \sum_{k<l}^{\prime} \sum_{m<n}{ }^{\prime \prime} f_{i j} f_{k l} f_{m n}+\ldots\right\} \Phi_{\mathrm{HO}}\left(\mathbf{r}_{1}, \ldots, \mathbf{r}_{N}\right)
\end{aligned}
$$

In this expression, the correlation function $f$ is defined as

$$
f(r)=\sum_{n=1}^{\infty} \mathcal{S}(n) 2\left[\frac{2^{n} n !}{(2 n+1) ! !}\right]^{1 / 2} L_{n}^{1 / 2}\left(\frac{1}{2} \alpha^{2} r^{2}\right)
$$


and the sums over single pairs, double pairs, ..., reflect the combinatorics of the selection of annihilation operators for the contraction of the creation operators contained in $: \exp \left(S^{(1,2)}\right)$ : The primes mean that no repeated particle indices appear in the multiple sums. The dots in Eq. (33) indicate up to $N / 2$ sums.

In Fig. 2 are displayed the pair correlation functions $f(r)$ for the solutions considered in Fig. 1. With the corresponding wave function given in Eq. (33) we have computed the expectation value of the Hamiltonian by means of the Monte Carlo method with a Metropolis sampling, and the reader should be aware that the sampling with a wave function like (33) is very costly in terms of computation time.

The simplest (and test) case corresponds to $N=4$. There we have not found spurious solutions, and the variational Monte Carlo (VMC) estimate for the energy corresponding to the lowest-energy TICC2 solution is $-25.55 \pm 0.05 \mathrm{MeV}$, in a very good agreement with the normal TICC2 value, which is displayed in Table 1.

The test has also been carried out for the normal and the spurious solutions of $N=16$. For the solid line solution the VMC value is $-1207 \pm 16 \mathrm{MeV}$, in good agreement with the normal TICC2 value, which is displayed in Table 1. However, the VMC value for the long-dashed solution turns out to be $-661 \pm 171 \mathrm{MeV}$, with a very large variance, and very different from the corresponding TICC2 value of $-1638 \mathrm{MeV}$. This is, in our mind, a definitive argument to consider the long-dashed line of Figs. 1-2 as a spurious solution, which should be ruled out. It is very interesting the agreement between TICC2 and TICC2-VMC calculations, and it is particularly stimulating if we realize that the TICC2 method is not variational. The closeness of both results means that TICC2 determines appropriately the energy.

The non-variational character of the $\operatorname{SUB}(n)$ truncations of CCM should not be considered as a drawback. Variational methods are very popular in many-body calculations, but the only advantage of using them, apart from obtaining an upper bound, is just to secure an improvement of the ground-state energy when the Hilbert space is enlarged. On the other hand, the hierarchical structure of the $\operatorname{SUB}(n)$ truncation has some similarities with perturbation theory, in the sense that the exact value is not monotonically approached. Both methods may be quite far away from the exact (and unknown) ground-state energy: in Jastrow case because of using an inadequate correlation, and in CCM because of a too early truncation.

It is worth mentioning that the problem of spurious solutions of the CCM method is one of the open questions related to this theory. In some simple cases [10], it has been possible to study the full map of solutions, but nevertheless these studies do not help too much in understanding the real origin of the spurious solutions. Here, the claimed advantage of the CCM, i.e. its non-linear character, turns out to be also a serious drawback.

Once the TICC2 solution has been properly identified, it is time to comment on the physical results, going back to Table 1. To this end, we should have in mind that a nuclear bosonic system with the central S3 interaction tends to collapse when the number of particles $N$ increases, so that it magnifies the role of the correlations. In Table 1 we can see that going from TICI2 to TICC2 results in an increasing gain of energy, ranging from $0.18 \mathrm{MeV}$ for $N=4$ to $963.24 \mathrm{MeV}$ for $N=40$. The importance of the non-linear terms contained in TICC2 increases with the number of particles. 
It is also interesting to compare with the results provided by an hybrid ansatz which includes pair correlations as well as hyperradial excitations in a TICI2 scheme [11, 12]. The combination of two-particle plus hyperradial correlations is the base of the hyperspherical harmonics description largely used in few-body systems [13, 14]. The entry TICI2+HR in Table 1 shows the results obtained by minimizing the Hamiltonian with respect to the correlation function $f(\rho, r)$, where $\rho$ is the hyperradius. Such an ansatz is an obvious generalization of the TICI2 one. The comparison shows that the gain in energy due to hyperradial excitations is relatively small. TICI 2 results change in less than $2 \%$ when hyperradial correlations are included. The gain due to TICC2 is much more important.

Finally, to complete this analysis it is important to compare our results with those obtained using a Jastrow correlation function. The entry J-VMC in Table 1 shows the ground-state energies determined by a variational Monte Carlo procedure. For $N=16$ and $N=40$ a rough determination of the ground-state energy was made 11 using a simple Jastrow correlation function $f_{J}(r)=1+a \exp \left(-b r^{2}\right)$. For $N=4$ we quote the Jastrow result given in Ref. [6]. We can see that our TICC2 results are still much less bound than the Jastrow variational lower bound. This reveals the importance of three-body and higher order correlations.

\section{$5 \quad$ Final comments}

In this paper we have presented a way of determining the ground-state energy and wave function of a system of bosons described in terms of the translationally invariant formulation of the coupled-cluster method. The analysis has been limited to the case of two-body correlations (TICC2 approach), and has been carried out by using Fock space techniques. The correlation amplitudes satisfy a non-linear coupled set of equations, which has been explicated here, and has been solved for light and medium bosonic nuclear systems, considering the S3 semi-realistic interaction of Afnan and Tang.

To conclude we would like to mention the required further steps and the pending questions to be solved on line. The first obvious extension of TICC2 is to consider SUB(3) and presumably SUB(4) truncation, as it is suggested by the comparison with Jastrow results. Here the main problem to face up is to find a unique way of describing the translationally invariant cluster operators, both in coordinate and in Fock spaces. One may propose that the three-body operators should be a mixture of $1 \mathrm{p}-1 \mathrm{~h}, 2 \mathrm{p}-2 \mathrm{~h}$ and $3 \mathrm{p}-$ $3 \mathrm{~h}$ excitations, and the main questions are how to find a unique description (i.e. the object equivalent to $\mathcal{S}(n)$ of $\mathrm{SUB}(2)$ ), and its relation to the characterizing quantum numbers.

In previous studies restricted to light systems (or to heavier nuclei in the TICI2 approximation) the advantages of the coordinate representation have been stressed with regard to the actual computations and with respect to the richness of the correlation operators (i.e. the easy way to introduce spin and isospin dependence when dealing with true nuclei). The form of the wave function is known [see Eq. (33)], and it seems simpler to determine a single function $f(r)$ instead of a large number of numerical amplitudes $\mathcal{S}(n)$. The still open problem is to transform the Schrödinger equation with the ansatz (30) into an appropriate integro-differential equation for the two-body correlation function.

The coordinate representation of the $\operatorname{SUB}(2)$ truncation of CCM is quite similar to the 
familiar Jastrow form with only two-body correlations, where the model state is correlated by the action of the operator $\prod_{i<j}\left[1+f\left(r_{i j}\right)\right]$. The expansion of this product generates all terms appearing in Eq. (33) and also additional terms which go beyond the independentpair character of that equation. It is worth noting that a form like Eq. (33) was used time ago 15] to obtain hypernetted-chain equations for the case of state-dependent correlations. Moreover, the main advantage of the Jastrow ansatz with respect to the SUB(2) truncation, particularly in the case of strong interactions, is its ability to tame the potential for all nucleon pairs. It has been suggested [16] to use this formal analogy to generate other truncation schemes for CCM which incorporate a Jastrow-like behaviour, and specially adapted to strongly short-distance repulsive interactions.

The last open question is to find a TICC2 formulation for fermionic systems. Here again we expect serious algebraic difficulties related to the structure of the reference state, with several occupied shells and the corresponding complexity of the $S^{(1,2)}$ operator. In our opinion, the formulation in coordinate space will simplify the problems related to the fermionic statistics.

\section{Acknowledgements}

This work was partially supported by DGICyT (Spain) grant PB92-0820. I.M. and M.P. acknowledge the DGICyT (Spain) and ICI (Spain), respectively, for a fellowship.

\section{References}

[1] F. Coester, Nucl. Phys. 7 (1958) 421.

[2] F. Coester and H. Kümmel, Nucl. Phys. 17 (1960) 477.

[3] H. Kümmel, K.H. Lührmann and J.G. Zabolitzky, Phys. Rep. 36 (1978) 1.

[4] R.F. Bishop, Theor. Chim. Acta 80 (1991) 95.

[5] R.F. Bishop, M.F. Flynn, M.C. Boscá, E. Buendía and R. Guardiola, Phys. Rev. C 42 (1990) 1341.

[6] R.F. Bishop, E. Buendía, M.F. Flynn and R. Guardiola, J. Phys. G: Nucl. Part. Phys. 18 (1992) 1157.

[7] R. Guardiola, I. Moliner, J. Navarro, R.F. Bishop, A. Puente and N.R. Walet, Nucl. Phys. A 609 (1996) 218.

[8] T.A. Brody and M. Moshinsky, Tables of Transformation Brackets for Nuclear ShellModel Calculations (Gordon and Breach, New York, 1967).

[9] L.R. Afnan and Y.C. Tang, Phys. Rev. A 175 (1968) 1337.

[10] R.F. Bishop and M.F. Flynn, Phys. Rev. A 38 (1988) 2211.

[11] R. Guardiola, I. Moliner and J. Navarro, Phys. Lett. B 383 (1996) 243. 
[12] R. Guardiola, I. Moliner and J. Navarro, Condensed Matter Theories, vol. 11, ed. E.V. Ludeña, P. Vashishta and R.F. Bishop (Nova Science, New York, 1996) p. 485.

[13] M. Fabre de la Ripelle, Ann. Phys. (NY) 147 (1983) 281.

[14] S. Rosati, M. Viviani and A. Kievsky, Few-Body Systems Suppl. 8 (1995) 21, and references therein.

[15] J.C. Owen, Ann. Phys. 118 (1979) 373.

[16] R.F. Bishop, Condensed Matter Theories, vol. 10, ed. M. Casas, M. de Llano, J. Navarro and A. Polls (Nova Science, New York, 1995) p. 483. 
Table 1: Binding energies (in $\mathrm{MeV}$ ) of $N$ bosons using the $\mathrm{S} 3$ interaction.

\begin{tabular}{lccc}
\hline & $N=4$ & $N=16$ & $N=40$ \\
\hline TICC2 & -25.49 & -1234.86 & -8456.55 \\
TICI2 & -25.31 & -1130.94 & -7493.31 \\
TICI2 + HR & -25.99 & -1145.11 & -7555.12 \\
J-VMC & -27.15 & $-1403 \pm 1.5$ & $-9570 \pm 7$ \\
\hline
\end{tabular}

\section{Figure captions}

Fig. 1. Energies (in MeV) of two selected TICC2 solutions (solid and long-dashed lines), plotted as a function of the number $N_{\max }$ of equations considered in the system (30). The TICI2 solution is also displayed as the short-dashed line.

Fig. 2. Correlation functions $f(r)$ as defined in Eq. (34) for the solutions of Fig. 1. 

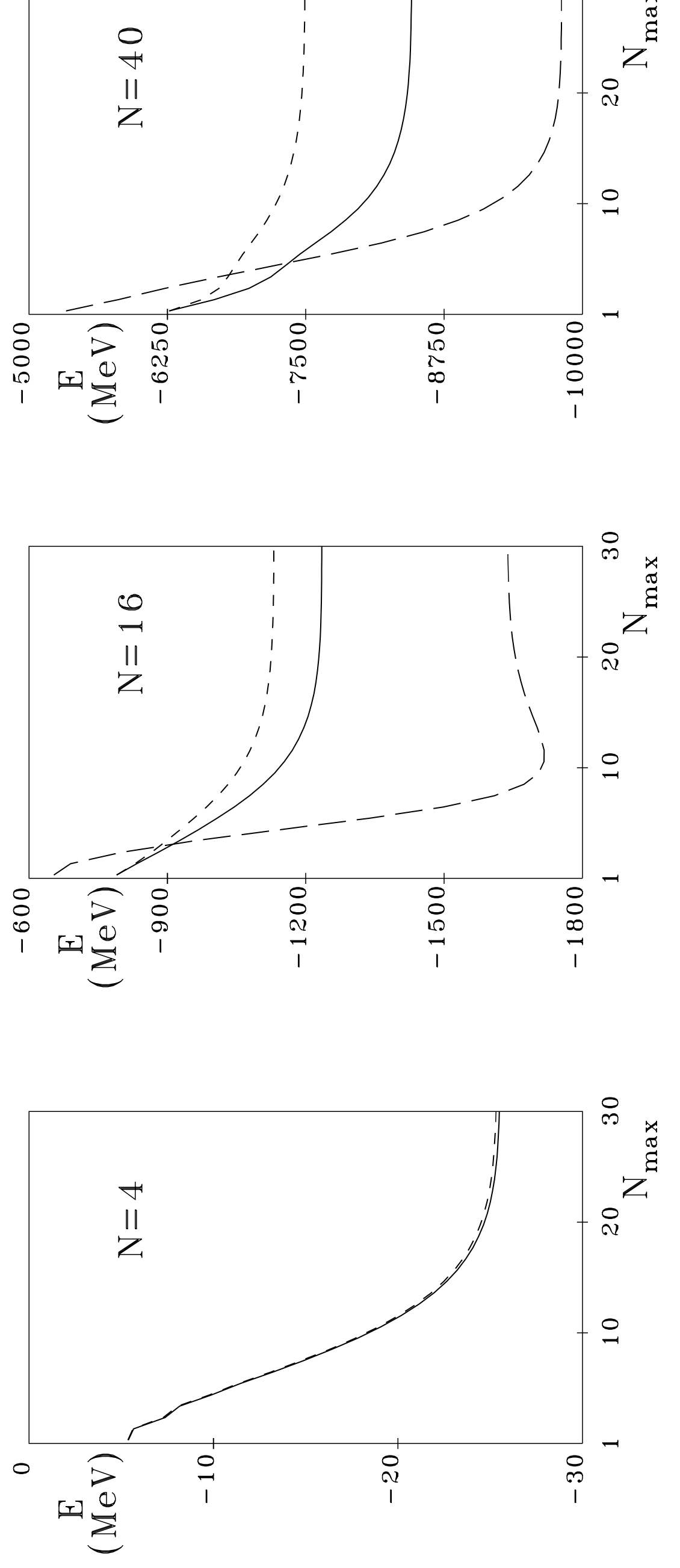
